\title{
Estrategias de Merchandising: un análisis de su efectividad para la atracción de nuevos clientes
}

\section{Merchandising Strategies: an analysis of their effectiveness in attracting new customers}

Freddy Verastegui Tene ${ }^{\mathrm{i}}$ Universidad César Vallejo fverasteguit@ucvirtual.edu.pe

Autor corresponsal.

Escuela de Marketing y Dirección de empresas, Facultad de Ciencias Empresariales, Uni-

Jorge Vargas Merinoi Universidad César Vallejo

jmerino@ucvvirtual.edu.pe versidad César Vallejo, Av. Alfredo Mendiola 6232. Los Olivos-Lima, PERUं

\section{Resumen}

Las técnicas del merchandising tienen la finalidad de atraer nuevos clientes y que estos realicen compras estratégicas dentro de los diferentes puntos de venta, incluso en el mundo digital. Sin embargo, muchas organizaciones continúan haciéndose la siguiente pregunta: ¿Qué tan efectivas pueden ser las técnicas de merchandising? Por lo cual, el objetivo de este artículo será discernir a través de la revisión de diferentes artículos científicos si esto se cumple en los diferentes contextos analizados. Se consultaron 132 artículos utilizando diversas bases de datos científicas, seleccionándose 56 que pertenecen a revistas arbitradas de Ebsco, Redalyc, Scopus, ProQuest y Google Academic. El merchandising de visualización, presentación, gestión, seducción y digital, permite lograr resultados muy favorables, quedando demostrado, por tanto, su efectividad, yya no solo el aplicable a tiendas físicas, sino a los espacios digitales, garantizando atracción de los nuevos clientes y ascensos sustanciales de las ventas de cualquier empresa. Finalmente, es importante concluir que los negocios que empezaron a actualizarse y adaptarse al nuevo modelo del merchandising digital, han tenido un fuerte incremento de ventas, nuevos clientes y expansión a distintos públicos, figura quizá forzada por el gran agente disruptivo del COVID-19; que sin duda llegó para quedarse.
INFORMACIÓN ARTICULO

Recibido: 14 de Julio 2020

Aceptado: 6 de Noviembre 2020

Palabras Claves:

COVID-19

Marketing digital

Merchandising

Merchandising digital

Visual merchandising 


\begin{abstract}
Merchandising techniques have the purpose of attracting new customers and that they make strategic purchases at the different points of sale, even in the digital world. However, many organizations continue to ask the question: How effective can merchandising techniques be? Therefore, the objective of this article will be to discern through the review of different scientific articles if this is true in the different contexts analyzed. 132 articles were consulted using various scientific databases, selecting 56 articles from peer-reviewed journals in Ebsco, Redalyc, Scopus, ProQuest and Coogle Academic. The visualization, presentation, management, seduction and digital merchandising allows to achieve very favorable results, its effectiveness is therefore demonstrated, and not only applicable to physical stores, but also to digital spaces, guaranteeing attraction of new customers and substantial increases in the sales of any company. Finally, it is important to conclude that the businesses that began to update to the new digital merchandising model have had a strong increase in sales, new customers and expansion to different audiences, perhaps forced by the great disruptive agent of COVID-19, but without certainly here to stay.
\end{abstract}

ARTICLE INFO

Received: 14 July 2020

Accepted: 6 November 2020

Keywords:

COVID-19

Digital marketing

Digital merchandising

Merchandising

Visual merchandising

\section{INTRODUCCIÓN}

Para Pérez, Pérez y Cristo (2018), el merchandising es una técnica eficiente en la interacción de la satisfacción de los clientes en el momento de compra de productos o servicios ofrecidos por una organización. Actualmente, las técnicas de comercialización tienen una gran importancia en los retail para lograr un mejor incentivo y satisfacción en la compra, a través del incentivo de venta (merchandising de seducción) y de la visualización (merchandising de gestión), entre otras técnicas.

Con lo mencionado anteriormente, podemos referir que el término en estudio tiene una gran amplitud conceptual, y se tiene una gran efectividad en los puntos de venta; sin embargo, las organizaciones creen que aplicar dichas técnicas, es símbolo solo de descuento y ofertas hacia los clientes. No obstante, dichas técnicas de comercialización tienen que ver con la presentación, lo visual, lo emocional y el desarrollo digital, haciéndose eficiente la comunicación al consumidor, el mismo que sin darse cuenta puede realizar más compras porque recibe la influencia directa de estas técnicas.

El merchandising es fundamental para todas las organizaciones, teniendo en cuenta que cada una de ellas tiene un enfoque distintivo, en este caso según Ahmed y Mujeeb (2017); también es importante puesto que ayuda a despertar el estímulo de los clientes en los puntos de venta, donde Cant, Wiid y Meyer (2016) consideran las percepciones éticas, simbólicas y culturales. En el presente artículo hablaremos de sus diferentes perspectivas 42 de ejecución, como el visual de juguetes que tiene una amplia categoría, por diseño, género, tamaño y costo. Sin embargo, se pueden manejar diferentes técnicas de comercialización para cada categoría y perspectiva de cliente.

Por otro lado, tenemos a Younes, Bragazzi y Pyatigorskaya (2019), quienes nos dicen que en el caso de una farmacia se aplicaron distintas técnicas de comercialización, entre ellas: disposición, POS-materiales y diseño de vitrinas, asimismo, se dio a conocer que el diseño de vitrinas tuvo un mayor impacto en el consumidor, mejoraron y añadieron algunos puntos, como la iluminación, el sonido y los aromas en el punto de venta.

Con relación a los párrafos anteriores, nos damos cuenta de que las técnicas de comercialización no solo pueden ser efectuadas para grandes organizaciones, sino que también para pequeñas empresas, como farmacias, jugueterías, tiendas de ropa, etc. Para ello se presentarán distintos casos de negocios con distintos enfoques que han tenido éxito en la aplicación de las técnicas en sus puntos de ventas y páginas web.

Por lo dicho anteriormente, las técnicas de comercialización tienen como finalidad atraer a nuevos clientes que realicen compras de forma estratégica dentro de los puntos de ventas y página web, a pesar de ello, muchas instituciones se realizan la siguiente pregunta: ¿Qué tan efectivas pueden ser las técnicas de comercialización?

Las instituciones que tienen puntos de ventas en distintos centros comerciales y otras tienen tiendas virtuales, buscan obtener una frecuencia cons- 
tante de la rotación de productos para lograr dicha meta, se aplican distintos tipos de técnicas, entre ellas están las estrategias de ventas. Sin embargo, varias empresas no le dan la importancia adecuada o no la saben aplicar correctamente, tanto en el sector del retail o en un escenario digital.

Esta revisión bibliográfica tiene como finalidad entender qué tan efectivas pueden ser las técnicas del merchandising y cómo han dado resultado en distintos contextos, aportando conocimientos fundamentales que puedan ayudar al desarrollo de futuras investigaciones. A continuación, se hará una breve presentación de los temas que se desarrollarán en esta investigación, teniendo como principal objetivo ofrecer una clara interpretación para el entendimiento de las técnicas. Para ello se recopiló información de autores científicos sobre las técnicas de comercialización y su efectividad en el mercado.

El primer tema a abordar es "El estado del arte del merchandising", donde se percibirá ¿Qué es el merchandising? y ¿Qué técnicas son las más usadas en el punto de venta o tienda digital?, para el segundo tema se enunciará "Merchandising: análisis de su efectividad desde diferentes enfoques de negocios", en este apartado se analizarán los distintos métodos de su aplicación en las distintas tiendas y categorías, y por último, el tema "Merchandising digital", en cual se enfocará netamente en la aplicación de las tiendas tradicionales y las tiendas virtuales, para saber cómo las organizaciones pueden aumentar sus ventas con el apoyo de estas técnicas en el mundo digital.

\section{MÉTODO}

La presente investigación es un artículo de revisión bibliográfica, que tiene por objetivo conocer y evaluar los hallazgos y lo informado por diversos autores en distintas bases científicas, además de actualizar los conceptos de una temática en evolución, como lo es el merchandising. Con el fin de seguir un orden en el proceso de revisión bibliográfica, se consideró la propuesta de Guirao, Olmedo y Ferrer (2008, citados por Acevedo-Gelves y Albornoz-Arias, 2018), quienes lo resumen en siete grandes etapas:

- Definición del problema y objetivos de la revisión

- Realización de la búsqueda de la bibliografía

- Consulta en diferentes bases de datos
- Establecimiento de las estrategias de búsqueda

- Especificación de los criterios de inclusión y exclusión de documentos

- Organización de la información, y

- Redacción del artículo

Con la revisión bibliográfica podemos recopilar datos e información que se presentan para la aclaración de un tema o conocer los distintos resultados obtenidos de las investigaciones (Ferreras, Rodero, García y Vega, 2016). En este caso podemos decir que, la metodología aplicada en esta investigación nos lleva a tener una amplia diversificación de información obtenida por los autores, para luego proceder a una selección con dicha información teniendo los parámetros requeridos, logrando un entendimiento con las teorías y de los distintos resultados sobre la investigación.

Es fundamental la revisión bibliográfica para la presente investigación, porque nos dará un mejor entendimiento sobre las técnicas del merchandising, teniendo una mayor comprensión del concepto, de su aplicabilidad y de su efectividad en las distintas empresas, además de conocer las perspectivas de dichas técnicas en el ámbito digital.

En el proceso de búsqueda se utilizaron diferentes terminologías o palabras clave, que aporten al hallazgo de los diferentes artículos requeridos; se aplicaron los siguientes términos: "Merchandising in retail", "Merchandising definition", "E-Merchandising", "Merchandising theories", "Merchandising strategy", "Merchandising aplicado en Latinoamérica", "Merchandising digital", "E-Comerce", "Merchandising psychology", "Mercancías", "Comercialización", "Merchandising cases", "Merchandising in Europe" "Merchandising en Perú" y "Merchandising in Asia", priorizando el idioma inglés y español (sin dejar de realizar algunas búsquedas en otros idiomas) y aplicadas en las bases de datos y buscadores Ebsco, Scopus, ProQuest, Redalyc y Google Academic.

Se consideraron preferentemente artículos científicos entre los años de 2013 y 2020; sin embargo, existen 8 artículos científicos, 7 de ellos expresados en tabla evolutiva de las teorías del merchandising para lograr un mejor entendimiento como definición en sus primeros años y, por último, 1 artículo que es del año 2006 que ayudó a enriquecer la información con respecto al merchandising digital. Por otro lado, los artículos seleccionados debian presentar un enfoque de conceptualiza- 
ción y descripción en cada una de las dimensiones mencionadas para los tres subtemas que aborda el presente artículo.

Como criterio de exclusión, se consideraron artículos científicos que no conceptualizaran lo referido a las técnicas del merchandising, es decir, del total de artículos buscados no han sido aceptados aquellos que no cumplieran los criterios referidos. La búsqueda quedó distribuida de la siguiente manera, Esbco: 43 artículos, Redalyc: 3 artículos, Scopus: 5 artículos, ProQuest: 51 artículos, Google Academic: 34 artículos, y aplicándose los criterios de inclusión y exclusión, quedaron 56 artículos.

En la Figura 1, los 56 artículos publicados en porcentajes según buscador; Ebsco: $32 \%$, Redalyc: 2\%, Scopus: 4\%, ProQuest: $38 \%$ y en Google Academic: 25\% (Ver Tablas y Figuras al final del documento).

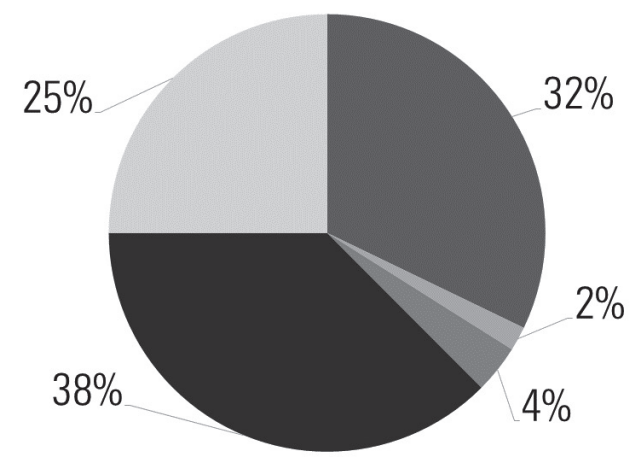

\#bsco $\square$ Redalyc $\square$ Scopus $\square$ ProQuest $\square$ Google Academic

Figura 1. Artículos citados según base de datos. Fuente: elaboración propia.

\section{DESARROLLO DEL TEMA Y DISCUSIÓN}

\section{Estado del arte del merchandising}

Wellhoff (2005) citado por Bonfanti (2014) nos menciona que el merchandising es un término de origen anglosajón que tiene como significado mercancía en acción, además de ello, tiene la terminación en inglés "ing", que lo convierte en un conjunto de técnicas que brindan a productos y servicios un rol importante en la venta, mejorando la visibilidad de la presentación y resaltando un marco que permita un potenciamiento en la rentabilidad.

Por su parte, Chang, Yan y Eckman (2014) mencionan que la técnica de comercialización mejora el atractivo de la tienda junto a su imagen percibida desde el punto de vista del consumidor, por lo que esta técnica da a los comerciantes un incremento de la atracción por el punto de venta, a la permanencia dentro de la tienda y con una mayor probabilidad de compra impulsiva, dándole al cliente una sensación de emoción y placer.

En tanto, Chkalova y Efremova (2019) expresan que se entiende como la tecnología de la gestión de ventas, enfocada a las empresas minoristas e incluso contiene variedad de tácticas. Asimismo, Westrup (2018), cree que estas técnicas son 44 actividades que se relacionan con el diseño del punto de venta, la visualización, diseño del espacio comercial, estanterías, vitrinas y exhibición de productos.

En complemento, Jain y Chheda (2020), comentan que las referidas técnicas de comercialización consisten en la presentación de productos en exhibiciones artísticas, maniquíes, prototipos de productos, entre otros, que ayudan a tener una mayor visualización atractiva y deseosa en percepción del consumidor, con un juego de colores, imágenes e interacciones en el punto de venta.

Widyastuti (2018), menciona que el merchandising visual causa un ambiente atractivo con respecto a la iluminación, el diseño, la comunicación visual y el aroma en la tienda, dando al cliente un impacto de atracción por las características mostradas, provocando un efecto de mayor circulación.

En este sentido, Gigoła (2016), lo consideran como técnica de comunicación del marketing, sirve para apoyar las ventas en sus diferentes puntos, por lo que el objetivo de esta técnica es fundamental, porque en el comercio no solo se trata de comunicar la información del producto (Duan, Mao y Huo, 2018), sino también de cómo atraer la atención del cliente, aplicando la estimulación en sus sentidos y 
la motivación de compra.

Kerfoot, Davies y Ward (2003) citados por Rizzi y Volpi, 2017) enuncian que las técnicas de mercancías tienen la capacidad de comunicar un producto y/o una marca a los consumidores, a través de una experiencia sensorial. Por lo que el mensaje debe ser entregado de forma psicológica y conductual positiva, para llevar como última instancia a la compra final.

Por otro lado, Pérez y cols. (2018), mencionan que las técnicas de mercancía también es un método eficaz, que logra los objetivos promocionales y el desempeño de favorecer la satisfacción de los clientes en los puntos de venta, con la aplicación de su tipología de seducción, el visual y el de presentación. Para ello, Little (2018), habla de que las técnicas de comercialización tienen una interactividad con la presentación del producto, la calidad de la imagen y la publicidad en el sitio web, y que estos son los más importantes para la experiencia del flujo del consumidor, asimismo, este autor también nos dice que, el desarrollo de un entorno con una alta resolución de la imagen en la tienda, la calidad, la interactividad y la publicidad atractiva, es clave para un buen merchandising visual, de atracción, la satisfacción y la compra.

De esta forma, lo que nos dan a entender los autores es que las referidas técnicas tienen una interactividad con el consumidor mediante el producto, servicio y establecimiento aplicado a los productos, estanterías, vitrinas, exhibiciones, espacios comerciales y en el punto de venta, haciendo que los consumidores y la imagen del retail incrementen en prestigio y ventas.

Para comprender mejor los conceptos del merchandising, se muestra una amplia variedad de definiciones de autores que nos explican cómo ha evolucionado su conceptualización a través de los años. En la Tabla 1 se observa que existen diversas definiciones, donde los autores plantean su conceptualización como técnicas en unos casos, otros lo toman como herramientas y también se ven como un complemento para generar experiencia y soporte en el ámbito promocional (Ver Tablas y Figuras al final del documento).

Tabla 1. Evolución de las definiciones de Merchandising

\begin{tabular}{|c|c|}
\hline Autor(es) / Año & Definición \\
\hline Buttle (1984) & $\begin{array}{l}\text { Explica que el merchandising analiza la rentabilidad como medio de garantía a una exposición previa a la compra hacia los consumidores } \\
\text { con material persuasivo o informativo, donde aplica } 5 \text { técnicas: manipulación del flujo de tráfico de la tienda, posicionamiento de estante; } \\
\text { asignación de espacio limitado en el estante entre reclamos en competencia; uso de material de punto de venta; y exhibiciones especiales. }\end{array}$ \\
\hline Buttle (1987) & $\begin{array}{l}\text { La comercialización es cualquier forma de promoción en la tienda, distinta a la venta personal y que está diseñada para activar el com- } \\
\text { portamiento de compra. Se le ha llamado "el silencioso vendedor ". Se ha convertido en un "arte científico" porque el comportamiento de } \\
\text { los clientes puede ser influenciado de manera predecible, y un arte porque se hace uso creativo de una batería de técnicas en constante } \\
\text { cambio. }\end{array}$ \\
\hline Little (1998) & $\begin{array}{l}\text { Nos dice que el merchandising es una actividad promocional realizado por un retail, para lograr un aumento en sus ventas. Donde aplican } 4 \\
\text { tipos de merchandising: Solo visualización; solo función; visualización y visión juntas; y recortes de precios no compatibles. }\end{array}$ \\
\hline $\begin{array}{l}\text { Kerfoot, Davies y Ward } \\
\text { (2003) }\end{array}$ & $\begin{array}{l}\text { Una forma de presentar el merchandising de manera efectiva para mejorar la conveniencia y efectividad de un producto es influir en el } \\
\text { comportamiento de compra del consumidor. }\end{array}$ \\
\hline Davies y Ward (2005) & $\begin{array}{l}\text { Explica que se utilizaron para limitar promociones, donde se aplica: color de mercancía; exhibición / presentación de mercancías; estilo de } \\
\text { los accesorios; los materiales utilizados en los accesorios; y accesorios e iluminación. }\end{array}$ \\
\hline $\begin{array}{l}\text { Polyxeni, Boutsouki y } \\
\text { Zotos (2010) }\end{array}$ & $\begin{array}{l}\text { Explica que el merchandising es importante en los puntos de venta, trabaja como una promoción, publicidad y el look de la tienda, mientras } \\
\text { que en la parte de páginas web se sincroniza con el e-mail para una mejor interactividad y seguimiento del consumidor. }\end{array}$ \\
\hline Liu (2011) & $\begin{array}{l}\text { El merchandising juega un papel importante en la mejora de los productos, apoya las marcas, aumenta el tráfico de clientes e incrementa } \\
\text { las ventas, agrega emoción visual a través de estrategias enfocado en la iluminación, en el entorno, la personalidad y la imagen en general }\end{array}$ \\
\hline $\begin{array}{l}\text { Garrido y González } \\
\text { (2015) }\end{array}$ & $\begin{array}{l}\text { Nos comentan que el merchandising no solo es la necesidad de incrementar la atracción, sino que también la necesidad de incentivo para } \\
\text { lograr un mejor resultado en la vista de los clientes. }\end{array}$ \\
\hline $\begin{array}{l}\text { Chowdhary y Hutson } \\
\text { (2018) }\end{array}$ & $\begin{array}{l}\text { Explica que el merchandising aumenta las ventas, con la aplicación de exhibiciones, exhibiciones dinámicas e iluminación, porque la aten- } \\
\text { ción entra por la iluminación y la visualización que se da en la tienda. }\end{array}$ \\
\hline $\begin{array}{l}\text { Sharma, Kedia, Yadav y } \\
\text { Mishra (2020) }\end{array}$ & $\begin{array}{l}\text { Nos dice que el merchandising se enfoca en la presentación de la tienda y su mercancía, en la cual hace que el consumidor tenga una } \\
\text { atracción y así generar ventas. }\end{array}$ \\
\hline
\end{tabular}

Nota: se observa que existen diversas definiciones con respecto al merchandising, donde algunos autores lo plantean como técnicas, otros lo toman como herramientas dentro del enfoque del merchandising visual, digital, de seducción y de presentación, asimismo, también lo ven como un complemento para generar experiencia y soporte en el ámbito promocional. Fuente: elaboracion propia. 
Se encuentran divergencias entre los diferentes autores, porque en un primer momento Buttle (1984) nos menciona que la técnica de comercialización limita el uso de la promoción, debido a que propone una mejor exhibición de sus productos con accesorios e iluminación que ayudan a tener un mayor impacto con el consumidor. Luego, tres años después Buttle (1987) nos dice que se convirtió en un vendedor silencioso, debido al impacto de las exhibiciones y sus complementos. Sin embargo, para Little (1998), indica que es una actividad promocional en las funciones de visualización, de función y en recortes de precios no compatibles. Aquí nos damos cuenta de que el merchandising al comienzo era asimilado como promoción, como un vendedor sigiloso; mas luego pasó a ser una actividad promocional.

Kerfoot, Davies y Ward (2003) indican que las técnicas de comercialización para que sean efectivas deben influir en el consumidor, en su comportamiento de compra; aunque, Davies y Ward (2005) explican que el merchandising se centra más en el producto, con la exhibición, accesorios e iluminación para una efectiva aplicación. Podemos inferir que el merchandising al pasar del tiempo mira al consumidor como objetivo a tratar, pero a la vez afirma que la manera eficiente es aplicando las exhibiciones, accesorios e iluminación a los productos.

En efecto, Polyxeni, Boutsouki y Zotos (2010), nos mencionan que las herramientas de comercialización trabajan de manera promocional, como también dando una esencia al punto de venta; también se refieren a la importancia del merchandising digital de las tiendas físicas y virtuales, con sus páginas web, redes sociales y los correos electrónicos para la interacción y comunicación con los consumidores fuera del punto de venta. Asimismo, para Liu (2011) la mercancía digital está enfocada en los consumidores frecuentes, donde la empresa brinda experiencia, descuentos y eventos exclusivos para dichos consumidores. En función a lo anteriormente mencionado, este tendrá un mayor crecimiento por el seguimiento y retención de los consumidores (Garrido y González, 2015).

Chowdhary y Hutson (2018) refieren que las técnicas de comercialización con la aplicación de exhibiciones dinámicas e iluminación en los productos tienen como efecto una mayor atracción de la tienda. Lo cual también es confirmado por Sharma, Kedia, Yadav y Mishra (2020) quienes indican que el merchandising se centra en la tienda y en la mercancía para lograr una venta.

46
Comparando los diversos autores, es posible decir que el merchandising tiene el objetivo de atraer a los clientes sin el apoyo de la promoción, aunque en sus principios pensaban que tenía un vínculo. No obstante, con el paso del tiempo ha evolucionado, mediante sus funciones como la visualización, presentación, gestión, seducción y la perspectiva digital, dándole un mejor entendimiento y efectividad a sus diferentes herramientas.

\section{Merchandising: análisis de su efectividad desde diferentes enfoques de negocios}

En tiempos de posmodernidad, Chowdhary y Hutson (2018) mencionan que la competitividad de las empresas es sumamente mayor y, sin duda, estas se están capacitando en la aplicación de técnicas de comercialización, en las cuales los minoristas están tratando de diferenciarse de los distintos puntos de venta, en su visualización de tienda, presentación de productos y en la seducción para atraer a clientes nuevos (Ver Tabla 2).

Con el paso del tiempo, las tiendas españolas han evolucionado en la aplicación de técnicas del merchandising, registrando un crecimiento considerable del 0,7\%, cuando la variación media en la comunicación y publicidad era del -9,9\%. El crecimiento del interés por esta muestra que hubo incidencia en las compras no planificadas, representando un incremento de entre el $46 \%$ y $70 \%$ de las compras totales (Infoadex, 2013 citado por los autores Garrido y González, 2015). Con lo mencionado, nos damos cuenta de que la influencia del merchandising ha aumentado, además de ello, causa una elección no decidida dentro de la tienda, demostrando que sí existe una efectividad en los puntos de venta.

En el caso de Liczmańska y Balcerak (2016), nos mencionan que la comercialización llegó a Polonia en los años noventa, donde las tiendas minoristas creían factible el uso máximo de espacio para atraer clientes; sin embargo, se dieron cuenta que no era suficiente; lo que generó la búsqueda de diferenciación en el mercado a través del sentido de la vista, así los minoristas empezaban a asociar sus tiendas con el tipo de cliente que querían acaparar, las tiendas iban mejorando su tienda, exhibición y atracción de nuevos clientes. El objetivo es consolidar a los minoristas, destacarse y desarrollarse.

Con respecto a las técnicas de comercialización, Garrido y González (2015), realizaron una prueba aplicando iniciativas de venta en los puntos establecidos para ello, con la presentación de pro- 
Tabla 2. Eficiencia de la aplicabilidad del Merchandising en distintos casos

\begin{tabular}{|c|c|c|}
\hline Autor(es) & Enfoque de negocio & País \\
\hline Chowdhary y Hutson (2018) & Competitividad de las empresas & U.S.A. \\
\hline Garrido y González, 2015). & Tiendas / Hipermercados & España \\
\hline Liczmańska y Balcerak (2016) & Evolución de los minoristas con el merchandising & Polonia \\
\hline Garrido y Gonzáles (2015) & Merchandising en espacios dentro del retail & España \\
\hline Hyun, Jung y Pauline (2015) & Tienda de moda & U.S.A. \\
\hline Dewhirst (2018) & Tienda de tabaco & Canadá \\
\hline Jiménez y Zambrano (2018) & Tienda textil & España \\
\hline Rodriguez, García y Bran (2017) & Tiendas físicas y online & España \\
\hline Kim y Takashima (2019) & Minoristas de supermercados & Japón \\
\hline Ramírez y Alférez (2014) & Cadena de supermercados & Colombia \\
\hline Cordova et al. (2020) & Mercado & Perú \\
\hline Yang y Lee (2019) & Comparación de varios negocios & Corea del Norte / Europa \\
\hline
\end{tabular}

Nota: se observa que existen diversas técnicas en la cual el merchandising es eficiente en distintos aspectos para distintas áreas y localización, logrando incrementar las ventas, ventas de otros productos, visualización de los productos y tienda, gestión de la tienda e iluminación. Fuente: elaboracion propia.

ductos en los extremos de los pasillos y la presentación de los productos en islas dentro de los principales pasillos; al momento de analizar la comparación, se verá también la influencia de los incentivos promocionales y qué es lo que puede causar esta combinación. El resultado de esta prueba fue efectiva para los dos casos de presentación de las islas en los pasillos principales y externos, además que los incentivos de venta le daban un enganche de compra, en este caso, podemos observar que el incentivo de venta complementa muy bien con la mercancía de exhibición (presentación).

En tanto, Hyun, Jung y Pauline (2015), nos dicen que las tiendas de moda aplicaron la comercialización visual al despertar la exploración de productos de marcas no conocidas, por otro lado, la tienda en estudio realizó una combinación de lo visual y lo cognitivo, logrando un conocimiento por medio de la percepción de los productos no exclusivos. Se examinó el impacto de la cognición visual con los productos de moda. El resultado de esta combinación es positivo, porque la comercialización le dio a la cognición visual actitudes de atracción de los productos con marcas no exploradas en la tienda y generó intenciones de compra.

Se analizaron distintos casos de negocios, donde es posible ver que la combinación de distintos fac- tores con el merchandising de visualización, presentación, gestión, seducción y el digital, puede lograr un resultado favorable en el incremento de ventas y la atracción de clientes.

Con relación a la efectividad de comercialización en el caso de una tienda de tabaco, Dewhirst (2018) indica sobre los hábitos de compra, que los consumidores potenciales tienen un producto seleccionado y conocen la trayectoria de la tienda, lo cual evita entorpecimientos con otros productos, para ello, se decidió aplicar el merchandising de gestión para generar rotación de productos variados y los productos al paso; remodelaron los ambientes donde los tabacos de mayor rotación iban en la parte final de la tienda, y los de menor rotación frente a ellos, además que los productos al paso como barra de chocolate, caramelos, chicles, etc., fueron reubicados en la caja para generar un impulso de venta con el tema psicológico del cuidado del higiene. Al cabo de tres meses se vio un ascenso del $13 \%$ de ventas de los productos al paso, y un ligero aumento de ventas de los productos con poca rotación.

Jiménez y Zambrano (2018) mencionan que los consumidores españoles no siempre suelen comprar de forma racional, pues en ocasiones gran parte de los consumidores se deja llevar por sus emociones. Por lo que en una tienda textil se pue- 
de retener más tiempo a los clientes, aplicando la técnica de comercialización de seducción, donde los clientes responden a la estimulación de los sentidos con el uso de la música para lograr una mayor permanencia, como también al uso de distintos colores para la diferenciación de secciones, por último, se potencia el aroma de la tienda para lograr un ambiente más envolvente, en donde la tienda adoptó el modelo de Hulten, Broweus y Van Dijk (2009), enfocado concretamente en la seducción visual, olfativa y sonora. El resultado obtenido fue una mayor permanencia en tiendas y un aumento doble en las ventas por el turno de la tarde.

Según Martínez y Aguado (2008) citado por Rodríguez, García y Bran (2017) la evolución de los formatos comerciales como en el caso de las ventas directas, se ha convertido en ventas electrónicas, estableciéndose una comunicación didáctica y continua con los consumidores, teniendo en cuenta que es la parte fundamental de las técnicas del e-merchandising o merchandising digital, en donde se traslada el enfoque de las tiendas físicas a las virtuales y la plataforma virtual tiene un gran espacio de información de producto, imagen, video y facilidad de pago. Sin embargo, el e-merchandising no deja de interactuar con las técnicas de seducción y visualización. De esta forma, se reduce costos de vendedores y establecimiento, lo que genera un crecimiento a la empresa.

En complemento, Kim y Takashima (2019) mencionan cómo las técnicas de comercialización pueden beneficiar a los minoristas de supermercados japoneses en las marcas privadas, donde la tienda aplica una cooperación del merchandising y las divisiones de la tienda, haciendo una mejora de las marcas privadas, fortaleciendo la competición contra las más reconocidas. Además de ello, el resultado de la mercancía tuvo una positiva relación entre la tienda y la marca privada. Sin embargo, los vendedores de la tienda se enfocan en las marcas reconocidas, en la cual no se explota todo el potencial puesto en el supermercado. En este caso, se pudo analizar que el supermercado estudiado realizó una aplicabilidad en sus productos de tienda, para tener una mayor ganancia y rotación de productos; sin embargo, descuidaron en los vendedores el priorizar dichos productos para que tengan un mayor impacto y aprovechar las técnicas de comercialización.

A continuación, dos casos de negocios en aplicación de técnicas de comercialización en Latinoamérica:
Ramírez y Alférez (2014) comentan sobre las principales cadenas nacionales de supermercados de la ciudad de Colombia, en donde los clientes cambian frecuentemente su decisión de compra por el efecto de las técnicas de comercialización visual, llegando incluso de un $28 \%$ a un $61 \%$ de clientes que cambian por la gran variedad de supermercados. Es por ello que se aplicó la comercialización visual con la finalidad de retener a los clientes en los supermercados, y para ello se aplicó una comunicación visual informativa y de persuasión al cliente, con las ofertas fuera del punto de venta para lograr un impacto de atracción, generando una inclinación por el supermercado analizado, además de una mayor circulación de clientes. El resultado fue favorable, un claro incremento en las ventas.

Córdova, Guerreros, Palomino y Ramos (2020), señalan que el merchandising visual tuvo una incidencia en la decisión de compra de los consumidores en tiendas minoristas regionales del Perú, tales como El Tambo y Huancayo. Se registró específicamente una influencia positiva en los factores culturales y sociales de la decisión de compra; sin embargo, fue negativo en los factores personales y psicológicos. Por otro lado, los puntos de venta no tuvieron una adecuada aceptación, porque los consumidores de ese sector no captaron la interacción de la comercialización visual.

Analizando la efectividad en las aplicaciones de las técnicas de comercialización en los diferentes negocios, podemos decir que han tenido un resultado positivo, además, Yang y Lee (2019) dicen que muchos negocios acompañaban la comercialización con otras herramientas o añaden incentivos para tener un mayor resultado, lo que repercute con mayor incidencia en los clientes nuevos.

\section{Merchandising digital}

En este último subtema se observará la función de la mercancía digital en la aplicación en tiendas digitales y en cómo influyó en ellas, también se verán las otras funciones respecto al e-merchandising. Para Crutsinger, Forney, Brandon y Jackson (2006), los procesos tradicionales del merchandising aplican tres elementos: planificación, desarrollo y presentación de mercancías, por lo cual, estos elementos son esenciales en tiendas físicas; sin embargo, estos también son importantes, pero con un enfoque diferente en el e-merchandising, el cual está operando en tiempo real, 
es decir, 24/7. Por consiguiente, los consumidores necesitan información de datos de inventario en tiempo real, de allí su importancia (Ver Tabla 3).

Tabla 3. Merchandising digital

\begin{tabular}{|c|c|c|}
\hline Autor(es) / Año & Merchadising digital & País \\
\hline Crutsinger et al. (2006) & Proceso tradicional del merchandising & U.S.A. \\
\hline Jain (2019) & Función del merchandising & India \\
\hline Deputatova et al. (2018) & Función del merchandising & Europa \\
\hline Yaoyuneyong, Foster y Flynn (2014) & Elementos del merchandising & China \\
\hline Gowda y Chaudhary (2018) & Compra en línea & India \\
\hline Vak ulenko et al. (2019) & Compra en línea & India \\
\hline Sung et al. (2018) & Tienda virtual & Corea \\
\hline Nguyen et al. (2020) & Sitio web & U.S.A. \\
\hline Badzińska (2013) & Merchandising digital & Polonia \\
\hline Abrar, Zaman y Satti (2017) & Merchandising digital & Pakistán \\
\hline Aljukhadar et al. (2020) & Social media & India \\
\hline Sampaio et al. (2015) & Social media & Brasil \\
\hline Sopiyan (2020) & Merchandising visual & Indonesia \\
\hline Keist y Bruer (2016) & Portafolio digital & U.S.A. \\
\hline Ahmed et al. (2020) & Covid-19 Merchandising & U.S.A. \\
\hline Seetharaman (2020) & Covid-19 Merchandising & India \\
\hline Hanna et al. (2020) & Covid-19 Merchandising & U.S.A. \\
\hline Lazebnyk y Ivasenko (2020) & Covid-19 Merchandising & Ucrania \\
\hline Puente (2018) & Servicio gráfico & U.S.A. \\
\hline Krasonikolakis et al. (2018) & Plataforma digital & Rusia \\
\hline Leung, Sun y Bai (2019) & Redes sociales & Japón \\
\hline
\end{tabular}

Nota: se observa que el merchandising digital ha incrementado con el desarrollo del C-19, por lo cual las tiendas digitales tuvieron una evolución óptima. Fuente: elaboracion propia.

Además de ello, Jain (2019), menciona que también se pueden filtrar las opciones y realizar comparaciones rápidas de productos sin estar presentes en el punto de venta, dando una experiencia nueva con la compra. Aunque, para Deputatova, Pshenitsyna, Ilyashenko, Baskakov y Zvereva (2018) los consumidores se han puesto más exigentes con la información respecto a la talla o el tipo de diseño, asimismo, hay que tener en cuenta otras barreras como la adquisición inmediata y el tipo de pago.

Para Ha, Kwon y Lennon (2007, citados en Yaoyuneyong, Foster y Flynn, 2014), los elementos digitales del merchandising en las tiendas digitales y sitios web de venta de ropa, empezaron a emplearse de forma taxonómica con señales en línea, para así poder lograr un efecto positivo, porque en la introducción de la comercialización digital recién empezaba y ya tenía una gran aceptación en el mercado.

En similar línea enunciativa, Gowda y Chaudhary (2018), mencionan que la compra en línea en la India se ha convertido en una nueva forma de vida, se estima que el mercado en línea en la India crecerá 3.5 veces con un alcance de 175 millones en el 2020. Una de las primeras causas por los que los consumidores han elegido este medio, es que las empresas dan una mayor facilidad de visualización de productos, flexibilidad en la entrega y en el pago. Vakulenko, Shams, Hellström y Hjort (2019), se refieren a que varios retail en estos últimos años, se han caracterizado por el notable crecimiento del marketing digital, por lo que varios minoristas están aprovechando en su totalidad el 
manejo del internet llegando a más clientes, extendiendo su cadena de distribución, optimizando sus recursos y teniendo mayor utilidad.

Con respecto a los párrafos anteriores, nos damos cuenta de que la comercialización digital contiene los mismos elementos que el tradicional, por lo cual, la aplicación de estas técnicas se puede replicar; pero con un distinto enfoque, esto quiere decir que las tiendas digitales pueden aplicar algunas de las técnicas tradicionales como el de presentación, visualización y seducción, y aprovechar las ventajas en la información de los productos, en comparación al tradicional.

Con el pasar de los años, podemos darnos cuenta de que las tiendas virtuales han estado en una constante evolución para lograr una mayor satisfacción en sus clientes y acrecentar su imagen. En este sentido, la mercancía digital tiene más funciones por desarrollar con la imagen en la parte digital, por ello Sung, Park, Sin, Kim y Kim (2018) mencionan que las imágenes de los productos en las tiendas virtuales se hacen más difíciles de clasificar por categoría, siendo una tarea titánica realizar una selección por nombre y tipo de productos.

Por su parte, Nguyen, Gruber, Fuchs, Marler, Hunsaker y Hargittai (2020) indican que el merchandising no solo es aplicable en los puntos de venta, sino que también está relacionado con los sitios web y las tiendas virtuales, en las cuales la imagen del producto va más allá para su aplicación. Con respecto a estos autores, podemos inferir que hay un grado de concordancia en lo que se debe lograr con una minuciosa clasificación de categorías, por lo que al momento de que un consumidor quiera realizar una compra tendrá complicaciones, por ejemplo, un cliente quiere comprar una bicicleta, las plataformas digitales no tienen categorías exactas sobre el producto; sin embargo, está adjunto a otros productos como los patines, scooter y las movilidades eléctricas. Esto afectaría a la aplicación efectiva en las redes sociales.

Para el caso de la comercialización de seducción digital, un claro ejemplo sería la atracción de clientes a costa de la innovación. Aquí podemos mencionar a McDonald's quienes innovaron con una plataforma digital donde los consumidores piden sus productos de manera digital y ya no de la forma tradicional como venían haciendo distintas cadenas de comida rápida. Januszewski y Żyminkowski (2007) citados en Badzińska (2013) mencionan que usando merchandising digital, nueve de cada diez personas que visitan centros comerciales con tiendas virtuales, han destacado sus promociones en dicho ámbito. Sin embargo, para Abrar, Zaman y Satti (2017), las tres cuartas partes de los clientes de centros comerciales lo ven como visualizaciones instaladas, y como fuente de información de los productos y sus promociones.

Por su parte, Aljukhadar, Poirier y Senecal (2020), expresan que el social media genera imágenes de usuarios, lugares y productos, logrando captar la atracción de millennials, por lo cual Instagram, Snapchat, Facebook y Pinterest se basan en el consumo de imágenes, por ello, para Santini, Sampaio, Perin, Espartel y Ladeira (2015), los jóvenes estudiantes de una universidad de Brasil son compradores impulsivos por tiendas virtuales, porque contienen una fuerte sincronización con los perfiles del consumidor estudiantil, haciendo que los estudiantes tengan una atracción por la aplicación de la mercancía de seducción en la parte digital.

Sopiyan (2020), menciona que la comercialización visual en lo digital tiene como objetivo comunicar y educar a los clientes en la mejora de la imagen de la tienda o empresa, para lograr un positivo impulso de ventas, en donde se presenta los productos de forma didáctica e interesante, con imágenes, videos y secciones informativas. Por otro lado, la comercialización digital puede ser aplicado también en personas, por ejemplo, los docentes de las universidades de Buffalo State University y Wade Collage, que promocionan recursos enfocados en el merchandising digital para la creación de una cartera en línea, en la cual dan un valor al estudiante en el momento de postular a una empresa (Keist y Bruer, 2016). En la actualidad, los estudiantes que crean su propio portafolio deben tener claro que este portafolio digital es un soporte más del currículo y para ello deben imprimir y organizar información de sus trabajos estudiantiles e imágenes de asistencia a eventos, con la finalidad de destacar al momento de presentarse en alguna corporación.

Según Ahmed, Streimikiene, Rolle y Duc (2020) con el Covid-19 (C-19) se ha tenido un efecto aterrador, que ha intimidado a consumidores desde China hasta EE. UU., con lo cual el comportamiento de los consumidores ha sido de compras de pánico y compras impulsivas, debido al cierre de tiendas y al contagio. Gran parte de los almacenes de EE. UU. se ha convertido en una pesadilla por las extensas hileras de clientes en compras de 
desinfectantes, agua embotellada, leche, enlatados y papel higiénico. Seetharaman (2020), indica que a meses de iniciada la pandemia, las empresas han caído en crisis, algunas cerrando, otras tratando de sobrevivir y muy pocas buscando caminos estratégicos que estén con relación al [-19, empezando a vender virtualmente, empresas que no tenían Delivery o tiendas virtuales empezaron a implementarlas para poder sobrellevarlo. Hanna y cols. (2020), señalan que producto de la pandemia las personas empezaron a no asistir a los supermercados, tiendas o algún establecimiento y comenzaron a pedir Delivery o compras por internet con mayor frecuencia, sin duda una gran oportunidad por aprovechar.

En tanto, Lazebnyk y Ivasenko (2020) refieren a que los negocios de los restaurantes en Europa han sido fuertemente golpeados por consecuencias del C-19, para las cuales sugieren formas de superación según la necesidad y la conveniencia en la aplicación en las redes sociales de los distintos restaurantes, aplicando la comercialización de seducción y visualización para obtener un impacto de confiabilidad con el cliente, en donde aplican la opción del Delivery, tomando las medidas de precaución y desinfección de los pedidos.

Para concluir este subtema es importante mencionar a Puente (2018), quien se refiere a que con el nacimiento de los servicios gráficos el vendedor desapareció, por lo que los productos tuvieron que empezar a venderse por sí solos, es por ello que nació el merchandising digital con técnicas relacionadas con el marketing de distribución.

Krasonikolakis, Vrechopoulos, Pouloudi y Dimitriadis (2018) mencionan que las plataformas digitales deben lograr que los productos se muestren y se vendan según la necesidad del cliente, aplicando al máximo las redes sociales, como también, Leung, Sun y Bai (2019) postulan que las imágenes, videos e información, desarrollan la satisfacción de la necesidad del consumidor y la mejora en la rentabilidad en la empresa.

Podemos decir que, en la actualidad, alrededor del mundo este formato de trabajo de los negocios virtuales en sus diferentes rubros ha tenido que adquirir las técnicas de comercialización de los negocios tradicionales; sin embargo, los negocios que no se han podido adaptar o implementarlo a su punto de venta digital, han tenido una caída en las ventas. Por otro lado, los negocios que empezaron a actualizarse han tenido un fuerte incremento de ventas, nuevos clientes y expansión a distintos públicos, producto del gran agente disruptivo: el C-19.

\section{CONCLUSIONES}

El merchandising es fundamental para todas las organizaciones, teniendo en cuenta que cada una de ellas tiene un enfoque distintivo, ayuda a despertar el estímulo de los clientes en los puntos de venta, considerando las percepciones éticas, simbólicas y culturales de cada uno de ellos. La comercialización es una técnica de comunicación de marketing y sirve para apoyar las ventas en los diferentes puntos, físicos o digitales, su objetivo es fundamental porque no solo se trata de comunicar la información del producto, sino también de cómo atraer la atención del cliente, aplicando la estimulación en sus sentidos y la motivación de compra.

Se han analizado distintos casos de negocios, en donde podemos ver que la combinación de distintos factores con la comercialización visualización, presentación, gestión, seducción y digital, permite lograr resultados favorables. Sin duda, la efectividad del merchandising está demostrado, y ya no solo es aplicable a tiendas físicas, sino a los espacios digitales con perfecta aplicación, garantizando atracción de los nuevos clientes y ascensos sustanciales de las ventas de cualquier empresa.

La compra en línea se ha convertido en una nueva forma de vida, ofrece una mayor facilidad de visualización de productos, flexibilidad en la entrega y en el pago para los clientes. Es importante reconocer el notable crecimiento del marketing digital, por lo que varios minoristas están aprovechándolo para llegar a más clientes, extendiendo su cadena de distribución, optimizando sus recursos y teniendo una mayor utilidad. La comercialización digital contiene los mismos elementos que el tradicional, pero con un distinto enfoque de aplicación, se trata finalmente de aprovechar las ventajas en la información de los productos que brindan las plataformas digitales.

Podemos decir que el COVID-19 ha generado que el mundo empresarial gire a la adaptación, casi total, de sus modelos de negocios, adoptando el modelo digital o virtual en sus diferentes facetas, y con ello, se han adquirido las técnicas de comercialización digital; sin embargo, existen empresas que no logran adaptarse o implementar un punto de venta digital con facilidad, y han registrado una 
caída en ventas importante, aunque los negocios que empezaron a actualizarse en este nuevo modelo, han tenido un fuerte incremento de ventas, nuevos clientes y expansión a distintos públicos, una figura quizá forzada por el gran agente disruptivo que es la pandemia.

Finalmente, se puede concluir que es importante ampliar el debate hacia la comprobación de la cuantificación de los efectos del merchandising, sobre todo del digital, con la realización de investigaciones empíricas multivariadas en diferentes contextos y modelos de negocio.

\section{REFERENCIAS}

Abrar, K., Zaman, S. y Satti, Z. (2017). Impact of online store atmosphere, customized information and customer satisfaction on online repurchase intention. Clobal Management Journal for Academic y Corporate Studies, 7(2), 22-34.

Acevedo-Gelves, L. K., y Albornoz-Arias, N. (2019). Revisión teórica de las capacidades dinámicas empresariales. Revista cientifica Pensamiento y Gestión, 46(1), 262-283.

Ahmed, Q. B., y Mujeeb, S. M. (2017). Retail store impression and shopper's behavior-a study of prozone mall Aurangabad. Indian Journal of Commerce and Management Studies, 8(1), 64-68.

Ahmed, R. R., Streimikiene, D., Rolle, J-A, y Duc, P. A. (2020). The COVID-19 Pandemic and the Antecedants for the Impulse Buying Behavior of US Citizens. Journal of Competitiveness, 12(3), 5-27.

Aljukhadar, M., Poirier, A. y Senecal, S. (2020). Imagery makes social media captivating! Aesthetic value in a consumer-as-value-maximizer framework. Scholarly Journals, 14(3), 285-303.

Badzińska, E. (2013). Media interaktywne warunkiem skutecznej komunikacji społecznej. Nierówności społeczne a wzrost gospodarczy, 35(1), 24-41.

Bonfanti, A. (2014). A dilemma for retailers: How to make store surveillance secure and appealing to shoppers. In Handbook of research on retailer-consumer relationship development, 297-317. IGI Global.
Buttle, F. (1984) Merchandising. European Journal of Marketing, 20(18), 104-123.

Buttle, F. (1987). Can you afford to ignore merchandising? Management Decision, 25(6), 1417.

Cant, M. C., Wiid, J. A., y Meyer, A. (2016). SMEs: Do they follow a shotgun or rifle approach when it comes to target marketing? Problems and perspectives in management, 14(3), 504511.

Chang, H. J., Yan, R. N., y Eckman, M. (2014). Moderating effects of situational characteristics on impulse buying. International Journal of Retail \& Distribution Management, 4(42), 298-314.

Chkalova, O. V., y Efremova, M. V. (2019). Merchandising as an innovative technology for sales efficiency management. Economic Analysis: Theory and Practice, 18(2), 265-278.

Chowdhary, U. y Hutson, C. (2018). Textile testing and visual merchandising displays. Journal of Family and Consumer Sciences, 110(2), 29-36.

Córdova, G., Guerreros, S., Palomino, F. y Ramos, W. (2020). Impact of visual merchandising on the purchase decision of consumers from retail stores in central Peru. Management Science Letters, 10(11), 2447-2454.

Crutsinger, C., Forney, J., Brandon, L. y Jackson, R. (2006). Requisite competencies and skills for E-merchandisers: Developing an interdisciplinary undergraduate program. Journal of Family and Consumer Sciences, 98(2), 81-88.

Davies, B. J., y Ward, P. (2005). Exploring the connections between visual merchandising and retail branding. International Journal of Retail o Distribution Management, 7(33), 505-513.

Deputatova, Y., Pshenitsyna, Y., Ilyashenko, S., Baskakov, V. y Zvereva, A. (2018). Consumer behavior in the context of global economic transformations. European Research Studies, 21(2), 95-109.

Dewhirst, T. (2018). Package size matters: Tobacco packaging, retail merchandising and its influence on trial and impulse sales. Tobacco Control, 27(5), 600-602.

Duan, Y., Mao, Z., y Huo, J. (2018). Introduction of store brands considering product cost and shelf space opportunity cost. Mathematical Problems in Engineering, 1- 19. 
Ferreras, F., Rodero; T., García, H. y Vega, F. (2016). The systematic review of literature in LIS. In: Proceedings of the Fourth International conference on technological ecosystems for enhancing multiculturality, 16, 291-296.

Garrido, M. y González, B. (2015). Merchandising at the point of sale: differential effect of end of aisle and islands. Business Research Ouarterly, 18(1), 57-97.

Gigoła, M. (2016). Eksperyment jako metoda badania wpływu Visual Merchandisingu na skłonności zakupowe. Prace Naukowe Uniwersytetu Ekonomicznego we Wrocławiu, (459), 79-89.

Gowda, K. y Chaudhary, K. (2018). Analysis of online buying pattern of women consumers with reference to apparels in India. Academy of Marketing Studies Journal, 22(2), 1-10.

Hanna, M., Reuter, V., Ardon, O., Kim, D., Sirintrapun, S., Schüffler, P., Klaus, B., Sauter, J., Brogi, E., Lee, T., Xu, B., Bale, T., Agaram, N., Tang, L., Ellenson, L., Philip, J., Corsale, L., Stameñps, E., Friedlander, M.,..., Meera, H. (2020) Validation of a digital pathology system including remote review during the COVID-19 pandemic. Mod Pathol. 33, 2115-2127.

Hulten, B., Broweus, N. y Van Dijk, M. (2009). Sensory Marketing. Great Britain: Britain Palgrave Macmillan.

Hyun, P., Jung, J. y Pauline, S. (2015). How does visual merchandising in fashion retail stores affect consumers' brand attitude and purchase intention? The International Review of Retail, Distribution and Consumer Research, 25(1), 87104.

Jain, G. (2019). Marketing Strategies for E-commerce Business. AAYAM: AKGIM Journal of Management, 9(2), 30-33.

Jain, A., y Chheda, R. (2020). Impact of Visual Merchandising on Consumer Behaviour: A Case of Rcity Mall. Studies in Indian Place Names, 40(35), 882-886.

Jiménez, M. y Zambrano, E. (2018). Marketing sensorial: merchandising a través de las emociones en el punto de venta. Análisis de un caso. adComunica: revista científica de estrategias, tendencias e innovación en comunicación, (15), 235-253.
Keist, C. y Bruer, S. (2016). Online Portfolios: Marketing Apparel Merchandising Millennials Into Employment. Journal of Family and Consumer Sciences, 108(2), 54-57.

Kerfoot, S., Davies, B., y Ward, P. (2003). Visual merchandising and the creation of discernible retail brands. International Journal of Retail o Distribution Management, 31(2), 143-152.

Kim, C. y Takashima, K. (2019), Effects of retail organisation design on improving private label merchandising, European Journal of Marketing, 12(53), 2582-2603.

Krasonikolakis, I., Vrechopoulos, A., Pouloudi, A. y Dimitriadis, S. (2018). Store layout effects on consumer behavior in $3 \mathrm{D}$ online stores. European Journal of Marketing, 52(5), 1223-1256.

Lazebnyk, ।. y Ivasenko, М. (2020). МЕТОДОЛОГІЧНІ ЗАСАДИ ДОСЛІДЖЕННЯ СОЦІАЛЬНИХ МЕДІА У РЕСТОРАННОМУ БІЗНЕСІ ЯК ІНСТРУМЕНТУ ПРОТИДІї НАСЛІДКАМ COVID-19. Problemy Ekonomiky, 1, 152-158.

Leung, X., Sun, J. y Bai, B. (2019). Thematic framework of social media research: state of the art, Tourism Review, 3(74), 517-531.

Liczmańska, K. y Balcerak, D. (2016). Visual merchandising on the example of network shop Mohito. Acta Universitatis Nicolai Copernici. Nauki Humanistyczno-Spoleczne. Zarzadzanie, 43(2), 141-152.

Little, J. D. (1998). Integrated measures of sales, merchandising, and distribution. International Journal of Research in Marketing, 15(5), 473485.

Liu, J. (2011). Sports merchandising, publicity rights, and the missing role of the sports fan. Boston College. Law School. Boston College Law Review, 52(2), 493-516.

Nguyen, M., Gruber, J., Fuchs, J., Marler, W., Hunsaker, A., y Hargittai, E. (2020). Changes in Digital Communication During the COVID-19 Global Pandemic: Implications for Digital Inequality and Future Research. Scholarly Journals. 6(3), 1-6.

Pérez, L. M., Pérez, F., y Cristo, Y. (2018). Aplicación del merchandising de seducción y el merchandising visual en el Palenque de los. Revista Infociencia, 22(3), 1-12. 
Polyxeni, P., Boutsouki, C. y Zotos, Y. (2010). Quantity surcharges and consumer awareness in a new retail environment. International Journal of Retail y Distribution Management, 38(5), 320-340.

Puente, N. (2018). Effective presentation e-merchandising techniques. The importance of review the literature to improve the management of digital companies. Harvard Deusto Business Research, 7(2), 88-102.

Ramírez, C. y Alférez, L. (2014). Conceptual model to determine the impact of visual merchandising in making purchasing decisions at the point of sale. Pensamiento y Gestión, 36, 1-27.

Rizzi, A., y Volpi, A. (2017). RFID-enabled visual merchandising in apparel retail. International Journal of RF Technologies, 8(4), 213-231.

Rodríguez, A., García, X. y Bran, C. (2017). Los iWatch: el intenso flujo de microformatos de "periodismo de un vistazo" alimentan seis de los principales medios online. Revista Latina de Comunicación Social, 72, 186-196.

Santini, F., Sampaio, C., Perin, M., Espartel, L. y Ladeira, W. (2015). Moderating effects of sales promotion types. BAR-Brazilian Administration Review, 12(2), 169-189.

Seetharaman, P. (2020). Business models shifts: Impact of Covid-19. International Journal of Information Management, 54, 102-173.

Sharma, V., Kedia, B., Yadav, V. y Mishra, S. (2020). Tapping the potential space-positioning of private labels. Journal of Indian Business Research, 12(1), 43-61.

Sopiyan, P. (2020). Pengaruh Strategi Harga dan Visual Merchandising terhadap Keputusan Pembelian Online. Jurnal Inspirasi Bisnis dan Manajemen, 3(2), 193-205.

Sung, J., Park, S., Sin, S., Kim, Y. y Kim, Y. (2017). Deep learning-based product image classification system and its usability evaluation for the 020 shopping mall platform. The Journal of The Institute of Internet. Broadcasting and Communication, 17(3), 227-234.
Vakulenko, Y., Shams, P., Hellström, D. y Hjort, K. (2019). Online retail experience and customer satisfaction: the mediating role of last mile delivery. The International Review of Retail. 29(3), 306-320.

Westrup, L. (2018). Merchandising Gen X: The Singles Soundtrack Album (1992/2017). Film Criticism, 42(2), 88-103.

Widyastuti, P. (2018). Does visual merchandising, store atmosphere and private label product influence impulse buying? Evidence in Jakarta. Journal of Business and Retail Management Research, 12(3), 16-24.

Yang, S. y Lee, Y. (2019). Mid- to low-end fashion brand personality affects consumers' perceived quality, commitment, and loyalty. Social Behavior and Personality, 47(7), 1-14.

Yaoyuneyong, G., Foster, J. y Flynn, R. (2014). Factors impacting the efficacy of augmented reality virtual dressing room technology as a tool for online visual merchandising. Journal of Global Fashion Marketing, 5(4), 283-296.

Younes, B., Bragazzi, L. y Pyatigorskaya, N. (2019). Influence of Sales Promotion Techniques on Consumers' Purchasing Decisions at Community Pharmacies. Pharmacy, 7(4), 150.

\section{AGRADECIMIENTOS}

Un especial agradecimiento a mi padre Fredy Verastegui Bustamante y a mi madre Betty Tene Guerrero por su apoyo incondicional durante el proceso de la investigación, como también a mi asesor académico Jorge Alberto Vargas Merino por su dedicación y apoyo permanente durante el proceso de elaboración de este artículo, y a su vez por la confianza otorgada. 